\title{
Foreword to the special issue on pattern recognition and image analysis
}

\author{
Jaime S. Cardoso ${ }^{1} \cdot$ Xose M. Pardo $^{2} \cdot$ Roberto Paredes $^{3}$
}

Published online: 15 July 2017

(C) The Natural Computing Applications Forum 2017

The papers included in this special issue provide a snapshot of image analysis and pattern recognition research today. They are the very best of the Seventh Iberian Conference on Pattern Recognition and Image Analysis (IbPRIA 2015), held on 17-19 June, 2015, in Santiago de Compostela, Spain. The IbPRIA is an international conference co-organised by the Spanish AERFAI and Portuguese APRP chapters of the IAPR International Association for Pattern Recognition. IbPRIA 2015 attracted 141 papers from 36 different countries. After the reviewing process, 83 papers were accepted for presentation in the conference. A selection of the best scored and presented at the conference was invited to submit to this special issue a substantially extended and revised version of the conference paper, and the resulting papers were sent out for full review. The process, including required revisions, was in accordance with the standing editorial policy of Neural Computing and Applications, resulting in the final versions of the 10 papers accepted and appearing in this special issue.

Segmentation is a fundamental task in medical image analysis, from which clinical information can be extracted. A representative case is the evaluation of cardiac function

Jaime S. Cardoso

jaime.cardoso@fe.up.pt

Xose M. Pardo

xose.pardo@usc.es

Roberto Paredes

rparedes@dsic.upv.es

1 INESC TEC and Faculty of Engineering, University of Porto, Porto, Portugal

2 CiTIUS, Universidade de Santiago de Compostela, Santiago De Compostela, Spain

3 Universidad Politécnica de Valencia, Valencia, Spain from cardiac magnetic resonance data, which requires measuring the left ventricle (LV) volume. Santiago et al. address precisely this problem, proposing solutions based on active shape models (ASM) to segment the whole MR volume simultaneously, instead of dealing with each slice independently. Polygonal approximations of digital planar curves are another important problem in image processing, pattern recognition and computer graphics. The main goal is to provide a compact representation of the original curve with reduced memory requirements, preserving the important shape information. Carmona-Poyato et al. propose an efficient algorithm, using a two-step procedure based on Pikaz's method to obtain a suboptimal polygonal approximation, followed by the improved Salotti's method to settle in the final approximation.

Prototype generation techniques for data reduction in instance-based classification aim at creating new data out of the elements of a given set so as to lower memory requirements while precisely defining the decision boundaries. Although these methods are commonly used in statistical pattern recognition, they turn out to be quite challenging for structural data as the merging operations required cannot be as clearly defined as in the former approach. Calvo-Zaragoza et al. propose the use of dissimilarity space representations, which allow us to map structural data representations onto feature ones, to benefit from the advantages prototype generation methods depict.

The recent digitalization of huge amounts of historical handwritten documents has raised the interest for automated methods to add value to the preservation efforts of Culture Heritage institutions, to provide adequate access to the contents of the preserved collections of handwritten text documents. Toselli et al. consider two document processing applications, computer-assisted transcription of text images (CATTI) and Keyword Spotting (KWS), both 
employing metadata structures called word graphs (WG). The authors study the trade-off between WG size and performance in terms of effectiveness and efficiency of CATTI and KWS, showing that small, computationally cheap WGs can be used without losing the excellent performance achieved with huge WGs. Still in the task of KWS, Puigcerver et al. present a new approach based on smoothing the (null) scores of out-of-vocabulary keywords by means of the information provided by "similar" invocabulary words. González-Rubio et al. study an unsupervised inference procedure for phrase-based (PB) translation models based on the theoretically well-founded minimum description length (MDL) principle. The authors propose practical solutions to overcome the limitations of current MDL approaches when applied to natural language, boosting the quality of MDL PB models.The learning and detection of objects with multiple intra-class appearance modes is still challenging in uncontrolled conditions. Villamizar et al. tackle this problem with a simultaneous object detection and clustering using boosted random ferns and probabilistic latent semantic analysis. The proposed method provides a discriminative and multimodal classifier that automatically clusters the response of its randomized trees in function of the visual object appearance. The authors show the method is capable of detecting objects with diverse and complex appearance distributions in realtime performance.

Human activity recognition is an important area of computer vision research. Most of the applications require an automated recognition of high-level activities, composed of multiple actions of multiple persons. Pereira et al. address the topic of social semantic meaning in a welldefined surveillance scenario and propose new definitions of individual and group behaviour that consider environment context, a relational descriptor that emphasises position- and attention-based characteristics, and a new classification approach.
Alonso et al. address the problem of social circles detection, a special case of community detection in social network that is currently attracting a growing interest in the research community. The authors define different vectorial representations from both structural egonet information and user profile features. The authors show that, provided structural network information is incorporated and modelled in the right way, multi-assignment clustering can constitute a valid technique for social circles detection.

The availability of a variety of sensors for the acquisition of biometric traits significantly degrades the performance of biometric systems when biometric data, acquired in one domain (sensor or illumination), are matched against the data acquired in a different domain. Such cross-domain recognition problem includes the cases when the images in one domain represent the sensor-specific periocular images. Kandaswamy et al. analyse the problem of mismatch between training and testing data, in which a deep model is trained on a source task and the knowledge acquired is then totally or partially transferred to help in solving a target task.

Thank you

Finally, we wish to express our deep gratitude to all the authors who submitted proposals to this special issue, particularly for the hard work they put in preparing these versions. We would like to thank the reviewers for their thoughtful review of the manuscripts, providing valuable feedback to improve the works. We are also very grateful to the Editor-in-Chief of Neural Computing and Applications, John MacIntyre, for giving us the opportunity to publish this special issue. We hope that this issue provides useful information for further research in pattern recognition and image analysis. 